\title{
Avaliação do consumo alimentar durante o COVID-19
}

Assessment of food consumption during COVID-19

\section{Elton Bicalho}

elton.bicalho01@gmail.com

Bianka Baptista Tito Vieira

bianka.vieira.77@hotmail.com 


\title{
RESUMO:
}

O atual contexto da pandemia causada pelo covid-19 trouxe impactos diretos nos hábitos alimentares e na prática de exercícios físicos, podendo ser um fator para o agravamento de doenças crônicas. O objetivo do presente trabalho foi verificar as mudanças nos hábitos alimentares de praticantes de atividade física durante a quarentena. Foi conduzido um estudo descritivo, com praticantes de musculação. Foi enviado por meio de aplicativo um questionário para coletar dados referentes ao perfil da amostra, questões referentes aos hábitos de vida, condições de saúde, alimentação e modificações corporais. Mudanças substanciais foram percebidas na alimentação e na ingestão hídrica, e 90,5\% dos participantes relataram perceber mudanças corporais. Frente aos resultados obtidos foi possível verificar que o isolamento social foi responsável por uma diminuição na prática de atividades físicas e promoveu algumas mudanças nos hábitos alimentares.

PALAVRAS-CHAVE Pandemias; Infeções por Coronavirus; Exercício Físico; Ingestão de Alimentos.

\begin{abstract}
:
The current context caused by covid-19 has had a direct impact on eating habits and physical exercise, which may be a factor in the worsening of chronic diseases. This article's objective was to verify the changes in eating habits of practitioners of physical activity during covid-19. A descriptive study was conducted with weight training practitioners. A questionnaire was sent through an application to collect data related to the sample profile, questions related to lifestyle, health conditions, food and body changes. Substantial changes were perceived in food and water intake, and $90.5 \%$ of participants reported perceiving body changes. In view of the results obtained, it was possible to verify that social isolation was responsible for a decrease in the practice of physical activities and promoted some changes in eating habits.
\end{abstract}

KEYWORDS: Pandemics; Coronavirus Infections; Exercise; Eating. 


\section{Introdução}

De acordo com a Organização Mundial da Saúde (OMS, 2010), a inatividade física é descrita como um dos mais importantes fatores de risco para mortalidade. Os níveis crescentes dessa inatividade causam implicações no estado geral de saúde e na prevalência de doenças crônicas não transmissíveis (DCNTs), tais como obesidade, doenças cardiovasculares, hipertensão, diabetes, cânceres entre outras. Por outro lado, a prática regular de exercício físico tem papel importante na redução do desenvolvimento de DCNTs, sendo determinante para o aumento do gasto energia e, consequentemente, influenciando no balanço energético e no controle do peso corporal (OMS, 2010), trazendo benefícios tanto para a saúde quanto para a composição corporal, resultando em melhorias na qualidade de vida (ABREU et al., 2017; MANTOVANI et al., 2017).

Seja por motivos estéticos ou por saúde, é inegável o aumento da população praticante de exercícios físicos e, por conseguinte, preocupada com uma alimentação mais equilibrada, uma vez que a alimentação pode auxiliar na melhora da performance e no desempenho físico (ALMEIDA; BALMANT, 2017). Entretanto, existem fatores que podem trazer mudanças bruscas no estilo de vida da sociedade, como a atual pandemia mundial de COVID-19. A doença ocasionada pelo novo coronavírus é uma síndrome respiratória aguda, cujas complicações, morbidade e rápida transmissão global levaram à adoção de importantes medidas de saúde pública, como o distanciamento social e o confinamento das populações dentro de suas casas (DI RENZO et al., 2020; FERNÁNDEZ-ARANDA et al., 2020). O confinamento da população trouxe impactos diretos nos hábitos alimentares e na prática de exercícios físicos, limitando atividades físicas e modificando a rotina. Muitas pessoas aumentaram o estoque de alimentos, pelo medo de sair de casa (DI RENZO et al., 2020; RUIZ-ROSO et al., 2020) e, pessoas confinadas tendem a ficarem entediadas e estressadas, susceptíveis à uma ingestão alimentar excessiva e compulsiva (RODRÍGUEZ-PÉREZ et al., 2020). Por outro lado, temos indivíduos vulneráveis a desordens alimentares, devido à recessão econômica e a consequente insegurança alimentar, além dos famosos "desafios de emagrecimento" e de "treinos em casa" que podem servir como gatilhos para as mesmas (WEISSMAN; BAUER; THOMAS, 2020).

O momento atual pode dificultar a manutenção de uma alimentação saudável e a prática regular de exercícios, porém, ainda é escasso o número de trabalhos que mensurem o atual contexto alimentar e de prática de exercícios por conta deste novo cenário. Diante deste contexto, o objetivo deste trabalho foi verificar as mudanças no comportamento e hábitos alimentares de praticantes de atividade física de alta intensidade durante a quarentena. 


\section{Métodos}

Foi conduzido um estudo descritivo com 21 praticantes de musculação. Para realização do trabalho, foram adotados os seguintes critérios de inclusão: idade mínima de 18 anos, indivíduos de ambos os sexos que praticavam musculação há pelo menos 1 ano e aceite por meio da assinatura do termo de consentimento.

Com o objetivo de coletar dados referentes ao perfil da amostra e questões referentes aos hábitos de vida, condições de saúde, utilização de ergogênicos, mudanças alimentares e corporais, foi aplicado um questionário elaborado pelos pesquisadores. Neste questionário também foi avaliada a autoperceção corporal através da Escala de Silhuetas de Kakeshita et al. (2009). O instrumento foi elaborado com o auxílio do aplicativo Microsoft Forms ${ }^{\circledR}$ e disponibilizado por meio de mensagem de texto, e a coleta de dados foi realizada durante o mês de Agosto de 2020 .

Foram realizadas análises de procedimentos clássicos por meio do software Microsoft Excel. Para a descrição do perfil da amostra e tabulação de alguns dados de relevância ao entendimento do estudo, foram utilizados procedimentos de estatística descritiva (média, desvio padrão, frequência relativa e frequência absoluta). $\mathrm{O}$ presente estudo foi aprovado pelo Comitê de Ética em Pesquisa do Centro Universitário de Volta Redonda - UniFOA, sob registro CAAE $n^{\circ}$ 37110820.0 .0000 .5237$.

\section{Resultados e discussão}

Ao total participaram 21 praticantes de musculação. A média de idade foi de $30,5 \pm 11,1$ anos com a maioria predominante de mulher $(\mathrm{n}=$ 16) que se declarou branca $(n=14)$, solteira $(n=$ 13), com renda mensal familiar de aproximadamente 2 a 5 salários mínimos $(\mathrm{n}=13)$, que cursou o ensino médio completo $(n=8)$ e trabalha $(\mathrm{n}=8)$. A tabela 1 apresenta a distribuição das variáveis sociodemográficas dos participantes da pesquisa. 
Tabela 1. Perfil sociodemográfico dos participantes da pesquisa

\begin{tabular}{|c|c|c|}
\hline Variável & $\mathrm{n}$ & $\%$ \\
\hline $\begin{array}{l}\text { Sexo } \\
\text { Feminino } \\
\text { Masculino } \\
\text { Total }\end{array}$ & $\begin{array}{c}16 \\
5 \\
21\end{array}$ & $\begin{array}{c}76,2 \\
23,8 \\
100\end{array}$ \\
\hline $\begin{array}{l}\text { Cor da Pele } \\
\text { Branca } \\
\text { Parda } \\
\text { Negra } \\
\text { Total }\end{array}$ & $\begin{array}{c}14 \\
3 \\
3 \\
20\end{array}$ & $\begin{array}{c}70 \\
15 \\
15 \\
100\end{array}$ \\
\hline $\begin{array}{l}\text { Estado Civil } \\
\text { Solteira(o) } \\
\text { Casada(o) } \\
\text { Viúva(o) } \\
\text { Total }\end{array}$ & $\begin{array}{l}13 \\
6 \\
2 \\
21\end{array}$ & $\begin{array}{c}61,9 \\
28,6 \\
9,5 \\
100\end{array}$ \\
\hline $\begin{array}{l}\text { Escolaridade } \\
\text { Ensino Fundamental Incompleto } \\
\text { Ensino Médio Incompleto } \\
\text { Ensino Médio Completo } \\
\text { Ensino Superior Incompleto } \\
\text { Ensino Superior Completo } \\
\text { Pós-graduação } \\
\text { Total }\end{array}$ & $\begin{array}{c}1 \\
1 \\
8 \\
6 \\
3 \\
2 \\
21\end{array}$ & $\begin{array}{c}4,8 \\
4,8 \\
38,1 \\
28,6 \\
14,2 \\
9,5 \\
100\end{array}$ \\
\hline $\begin{array}{l}\text { Ocupação } \\
\text { Trabalha } \\
\text { Estuda } \\
\text { Estuda e Trabalha } \\
\text { Desempregada(o) } \\
\text { Do lar } \\
\text { Total }\end{array}$ & $\begin{array}{c}8 \\
3 \\
6 \\
2 \\
2 \\
21\end{array}$ & $\begin{array}{c}38,1 \\
14,3 \\
28,6 \\
9,5 \\
9,5 \\
100\end{array}$ \\
\hline $\begin{array}{l}\text { Renda Familiar Mensal } \\
1 \text { SM* } \\
2-5 S M \\
>5 S M \\
\text { Não sabe } \\
\text { Total }\end{array}$ & $\begin{array}{c}2 \\
13 \\
5 \\
1 \\
21\end{array}$ & $\begin{array}{c}9,5 \\
61,9 \\
23,8 \\
4,8 \\
100\end{array}$ \\
\hline
\end{tabular}

* SM = salário mínimo nacional: $\mathrm{R} \$ 1.045,00$

Um estudo feito com residentes dos Estados Unidos da América (SHEN et al., 2020) realizado para explicar os efeitos do comer emocional e do estresse nas escolhas alimentares durante a pandemia contou com a participação de 800 adultos, dos quais $83 \%$ mulheres, $77,5 \%$ brancas, 64,4\% com ensino superior completo ou titulação superior, corroborando parcialmente com os achados do presente estudo. Outros estudos realizados no período da pandemia também verificaram maior prevalência do público feminino (CONSTANDT et al., 2020; HE et al., 2020; GÓRNICKA et al., 2020; DI RENZO et al., 2020; ZACHARY et al., 2020; CANCELLO et 
al., 2020; REYES-OLAVARRÍA et al., 2020; ANTUNES et al., 2020), o que pode ser explicado por uma ênfase da preocupação com a saúde feminina, visto que o cuidado não é considerado uma prática masculina, ou seja o comportamento social masculino tem estreita relação com possíveis agravos à saúde deste grupo (GOMES, 2003; PASCHOALICK; LACERDA; CENTA, 2006; GOMES; NASCIMENTO; ARAÚJO, 2007).
Sobre a saúde e os hábitos de vida, a maior parte ( $\mathrm{n}=18)$ alega ausência de doenças e não faz uso de nenhum medicamento $(\mathrm{n}=19)$. Com relação a prática de exercícios físicos, 42,9\% ( $\mathrm{n}=9)$ dos participantes relataram diminuição da frequência das atividades. Quanto a ingestão hídrica, 9 participantes afirmaram ingerir acima de 2 litros diários de água antes da pandemia, subindo para 10 participantes atualmente. A tabela 2 apresenta a distribuição das variáveis relacionadas saúde e aos hábitos de vida dos participantes da pesquisa.

Tabela 2. Saúde e hábitos de vida dos participantes dapesquisa

\begin{tabular}{l|c|c}
\hline \multicolumn{1}{c|}{ Variável } & $\mathrm{n}$ & $\%$ \\
\hline Doença & 3 & 14,3 \\
Sim & 18 & 85,7 \\
Não & $\mathbf{2 1}$ & $\mathbf{1 0 0}$ \\
Total & & \\
Medicamento & 2 & 9,5 \\
Sim & 19 & 90,5 \\
Não & $\mathbf{2 1}$ & $\mathbf{1 0 0}$ \\
Total & & \\
Frequência de Atividade Física (atual) & 7 & 33,3 \\
Manteve & 5 & 23,8 \\
Parou & 9 & 42,9 \\
Diminuiu & $\mathbf{2 1}$ & $\mathbf{1 0 0}$ \\
Total & & \\
Ingestão de Água (prévia) & 4 & 19 \\
1 L/dia & 3 & 14,3 \\
1-1,5 L/dia & 5 & 23,8 \\
1,5-2 L/dia & 9 & 42,9 \\
> 2 L/dia & $\mathbf{2 1}$ & $\mathbf{1 0 0}$ \\
Total & & \\
Ingestão de Água (atual) & 4 & 19 \\
1 L/dia & 4 & 19 \\
1-1,5 L/dia & 3 & 14,3 \\
1,5-2 L/dia & 10 & 47,7 \\
> L/dia & $\mathbf{2 1}$ & $\mathbf{1 0 0}$ \\
Total & &
\end{tabular}

$\mathrm{L}=$ litro

Fonte: Elaboração própria 
As mudanças alimentares relatadas durante a pandemia pelos indivíduos foram: aumento do consumo de refrigerantes $(9,5 \%)$, fast foods e alimentos ultraprocessados $(38,1 \%)$, além do aumento no volume das refeições $(4,8 \%)$. Algumas mudanças positivas também foram observadas, como maior consumo de frutas e hortaliças (19\%). Além disso, 28,6\% dos participantes não relataram mudanças na alimentação.

$\mathrm{Na}$ Bélgica uma pesquisa realizada por Constandt et al. (2020) com 13.515 pessoas teve como objetivo examinar os níveis e padrões de exercício de adultos durante o lockdown onde os participantes foram divididos em 2 grupos: high ative (grupo 1) $\mathrm{e}$ low active (grupo 2). O grupo 1, composto por 11.763 pessoas, demonstrou que $41 \%$ dos participantes mantiveram a frequência de atividade física, e destes que continuaram, $54 \%$ considerou ter mais tempo para se exercitar. Estes achados diferem dos resultados do presente estudo, uma vez que $42,9 \%$ dos participantes relatou uma diminuição na frequência de atividade.

Já na Polônia Górnicka et al. (2020) em pesquisa com 2.381 indivíduos buscou identificar os padrões de mudanças alimentares durante a pandemia e suas associações com mudanças no estilo de vida. Em seus resultados, foi possível verificar que 43,3\% diminuíram a frequência de atividades físicas, $49 \%$ aumentou o tempo de tela e $34,3 \%$ aumentou o consumo alimentar. Os resultados quanto à frequência de atividade física vão ao encontro do reportado pelos participantes deste estudo, sendo que o aumento do consumo alimentar foi uma das mudanças alimentares relatadas pelos participantes.

Ainda em relação à prática de exercícios, um inquérito online conduzido coma participação de 143 profissionais e estudantes de fisioterapia para quantificar o impacto do lockdown na prática de atividade física e gasto de energia foi conduzido por Srivastav, Sharma e Samuel (2020). Antes do lockdown o total de exercícios físicos praticados pelos participantes era de 7809,7 MET-min/semana e durante o lockdown esse tempo foi reduzido para 4135,7 MET-min/semana, mostrando uma redução no gasto de energia com a prática de exercícios.

Para determinar as mudanças no estilo de vida causadas pelo confinamento e analisar as associações, uma pesquisa com 700 chilenos foi conduzida por Reyes- Olavarría et al. (2020). Como principais resultados os pesquisadores descrevem que $45,3 \%$ dos participantes afirmaram que consumiam uma média de 3 a 5 copos de água/dia; 51,3\% mantiveram os hábitos alimentares anteriores a pandemia, porém, comendo em maior quantidade, além de $30 \%$ relatarem etilismo diário. Sobre a prática de consumo de fast foods e frituras, o consumo foi assinalado por $62,9 \%$ e $59,9 \%$ dos participantes, respetivamente, por pelo menos 1-2 vezes/semana. Sobre a atividade física, $51,2 \%$ dos homens e $58,7 \%$ das mulheres relataram diminuição na frequência. Tais resultados corroboram com o resultado presente no atual estudo em que a maior parte dos participantes relatou 
diminuição na frequência de atividade física, porém no atual estudo foram relatadas mudanças alimentares enquanto a maioria dos chilenos relataram manter os mesmos hábitos, e também em relação à ingestão de água os chilenos bebem cerca de $750-1250 \mathrm{ml} / \mathrm{dia}$ enquanto os participantes deste estudo bebem acima de 2L/dia.

Na Itália, Di Renzo et al. (2020) conduziram uma pesquisa online para analisar a relação entre os hábitos alimentares e o estado mental e emocional de 602 indivíduos. Os resultados apontaram que $44 \%$ dos participantes faziam dieta antes da pandemia, 14\% controlam a alimentação, 48,7\% comem quando ansiosos, $55,1 \%$ comem para se sentir melhor e $57,8 \%$ se sentem ansiosos com os hábitos alimentares atuais. Outra pesquisa realizada na Itália por Cancello et al. (2020) com 490 participantes que objetivava observar os efeitos do lockdown nas mudanças de estilo de vida e verificar seus determinantes. Antes do lockdown 270 indivíduos eram considerados ativos, sendo que deste total $68 \%(\mathrm{n}=184)$ reduziram a prática de exercícios. Sobre os hábitos alimentares, $34 \%$ perceberam uma melhora na qualidade da alimentação e $42 \%$ relataram ter aumentado o consumo alimentar. De maneira semelhante a redução na prática de exercícios foi observada no presente trabalho.

Em Portugal, Antunes et al. (2020) conduziram um estudo para caracterizar hábitos de vida, níveis de ansiedade e necessidades psicológicas durante a pandemia. Foram recrutados 1.404 adultos e como principais resultados os pesquisadores apresentam que
$54,8 \%$ dos participantes não tiveram aumento na frequência alimentar enquanto 31,6\% relataram aumento no consumo alimentar. No atual estudo dentre as mudanças alimentares relatadas o aumento do consumo alimentar foi uma destas, verificado em apenas $31,6 \%$ dos participantes do estudo de Antunes et al. (2020) e em $42 \%$ dos participantes do estudo de Cancello et al. (2020), e os hábitos atuais geram ansiedade em 57,8\% dos participantes do estudo de Di Renzo et al. (2020), fazendo com que $48,7 \%$ comam mais.

Sobre mudanças corporais que aconteceram durante a quarentena, a maioria dos participantes $(n=19)$ relatou ter percebido mudanças como ganho de peso, aumento de gordura corporal e perda de massa muscular desse total, apenas um participante relatou emagrecimento. Levando em conta a autoperceção de imagem corporal (figura 1), $23,8 \%$ dos participantes $(\mathrm{n}=5)$ afirmaram que sua silhueta antes da pandemia se parecia com a imagem 3 e, durante o período da pandemia, observou-se maior frequência de respostas para a imagem $6(19 \% ; n=4)$, o que nos apresenta um aumento de 3 pontos de ganho. A tabela 3 apresenta as variáveis relativas à mudança corporal e silhueta dos participantes da pesquisa. 
Figura 1. Escala de silhuetas de Kakeshita et al (2009).
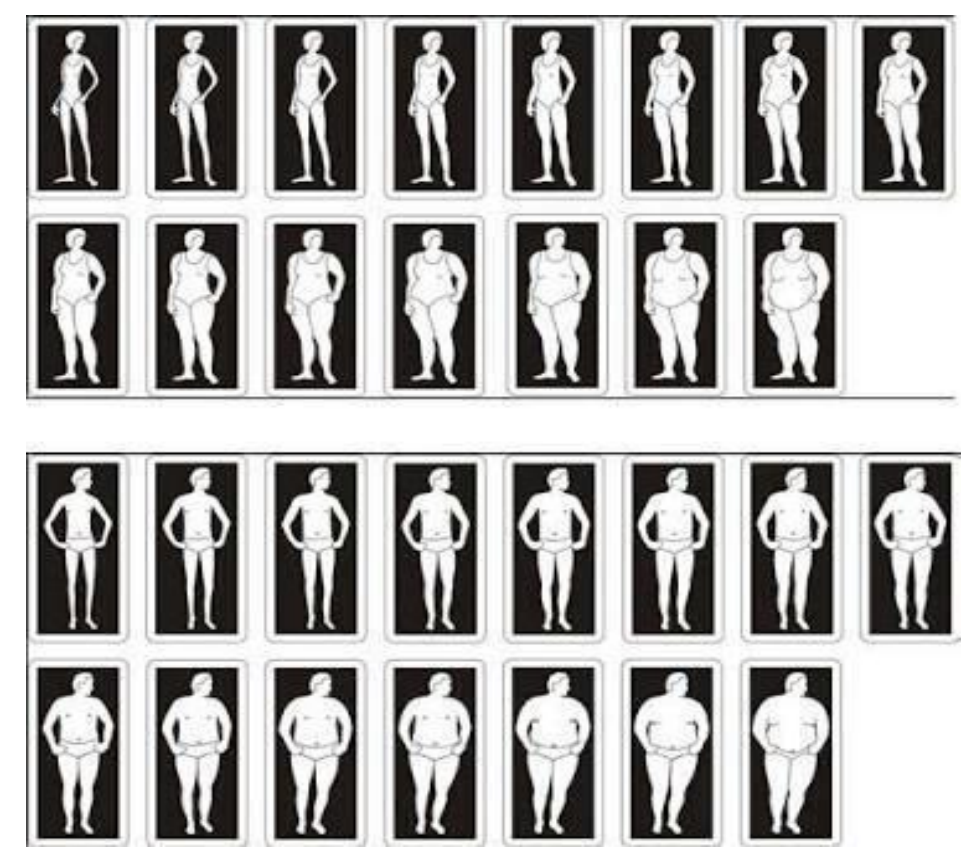

Fonte: Baseado em Kakeshita et al (2009)

Tabela 3. Mudanças corporais dos participantes da pesquisa

\begin{tabular}{c|c|c}
\hline Variável & $\mathrm{n}$ & $\%$ \\
\hline Silhueta (prévia) & & \\
Imagem 3 & 5 & 23,8 \\
Imagem 4 & 3 & 14,3 \\
Imagem 5 & 2 & 9,5 \\
Imagem 6 & 2 & 9,5 \\
Imagem 7 & 2 & 9,5 \\
Imagem 8 & 3 & 14,3 \\
Imagem 9 & 3 & 14,3 \\
Imagem 10 & 1 & 4,8 \\
Total & $\mathbf{2 1}$ & 100 \\
Silhueta (atual) & & \\
Imagem 3 & 3 & 14,3 \\
Imagem 4 & 2 & 9,5 \\
Imagem 5 & 3 & 14,3 \\
Imagem 6 & 4 & 19 \\
Imagem 8 & 2 & 9,5 \\
Imagem 9 & 3 & 14,3 \\
Imagem 10 & 1 & 4,8 \\
Imagem 11 & 1 & 4,8 \\
Imagem 12 & 2 & 9,5 \\
Total & $\mathbf{2 1}$ & $\mathbf{1 0 0}$ \\
\hline
\end{tabular}

Fonte: Elaboração própria 
De acordo com Demoliner e Daltoé (2020) o isolamento social causado pela quarentena pode predispor condições de estresse devido ao medo e ansiedade gerados, modificando hábitos alimentares prévios. $\mathrm{O}$ estresse prolongado neste período fará com que o corpo libere cortisol, aumentando a sensação de fome $\mathrm{e}$, consequentemente, um consumo maior ou mais frequente de alimentos, muitas vezes de maneira exagerada. Essa mudança de comportamento alimentar pode ser marcada também pelo estoque de alimentos com grande tempo de prateleira, geralmente industrializados. Os fatores citados, em conjunto com a redução da atividade física, são responsáveis por promoverem mudanças corporais, como o ganho de peso que pode aumentar o risco de desenvolvimento de obesidade e doenças associadas.

He et al. (2020) realizaram um estudo com chineses para avaliar a relação entre mudanças de peso, atividade física e estilo de vida durante a quarentena. De acordo com os resultados da pesquisa, a maioria dos participantes, independente do sexo, ganhou peso. Outro estudo com o propósito de quantificar o impacto da quarentena em fatores relacionados ao ganho de peso foi realizado online com 173 pessoas. Os autores descrevem que 59\% dos indivíduos alegaram ter mantido o peso estável, enquanto $22 \%$ relatou um aumento de aproximadamente $2-4,5 \mathrm{~kg}$ no peso corporal (ZACHARY et al., 2020).
Cancello et al. (2020) afirmam que dentre os 490 italianos participantes da pesquisa $42 \%$ alegaram aumento no consumo alimentar e 39\% ganharam peso. Em adição, ReyesOlavarría et al (2020) verificaram que na população chilena $25,6 \%$ dos homens e $38,1 \%$ das mulheres relataram ganho de peso. Dentre os estudos citados acima, todos verificaram ganho de peso nos participantes, mesmo que não tenham sido a maioria dos indivíduos como no atual estudo

\section{Conclusão}

Frente aos resultados obtidos por meio dos relatos dos participantes da pesquisa e dos trabalhos similares a este, foi possível verificar que o isolamento social foi responsável por uma diminuição na prática de atividades físicas e promoveu algumas mudanças nos hábitos alimentares dos participantes como o aumento do consumo de refrigerantes, fritura, fast foods, alimentos ultraprocessados, e aumento no volume das refeições, gerando, consequentemente, mudanças corporais, sendo a mais citada o ganho de peso. Ressalta-se que quando existe ganho de 'peso em excesso pode ocorrer os agravos à saúde associados ao sobrepeso e a obesidade, que por si já é um dos fatores de risco para a COVID-19.

É importante, portanto, que sejam estimulados os hábitos saudáveis para preservar a saúde dos indivíduos. Realizar exercícios em casa, estimular o preparo de refeições mais saudáveis e dar preferência aos alimentos in natura ou minimamente processados, além de 
reduzir o consumo de fast foods, refrigerantes e ultraprocessados são medidas que devem ser estimuladas, porém, de forma consciente, evitando o surgimento de transtornos alimentares ou distúrbios de comportamento relacionados a imagem corporal. 
ABREU, ES. et al. Hábitos nutricionais pré-treino de praticantes de ginástica do projeto de extensão PROGINC-UECE. Revista Brasileira de Nutrição Esportiva, v. 11, n. 62, p. 118-125, 2017.

ALMEIDA, CM.; BALMANT, BD. Avaliação do hábito alimentar pré e pós-treino e uso de suplementos em praticantes de musculação de uma academia no interior do estado de São Paulo. Revista Brasileira de Nutrição Esportiva, v. 11, n. 62, p. 104-117, 2017.

ANTUNES, R. et al. Exploring Lifestyle Habits, Physical Activity, Anxiety and Basic Psychological Needs in a Sample of Portuguese Adults during COVID-19. Int. J. Environ. Res. Public Health, v. 17, n. 4360, p. 1-13, 2020.

CANCELLO, R. et al. Determinants of the Lifestyle Changes during COVID-19 Pandemic in the Residents of Northern Italy. Int. J. Environ. Res. Public Health, v. 17, n. 6287, p. 1-13, 2020.

CONSTANDT, B. et al. Exercising in Times of Lockdown: An Analysis of the Impact of COVID19 on Levels and Patterns of Exercise among Adults in Belgium. Int. J. Environ. Res. Public Health, v. 17, n. 4144, p. 1-10, 2020.

DEMOLINER, F.; DALTOÉ, L. COVID-19: nutrição e comportamento alimentar no contexto da pandemia. Perspectiva: Ciência e Saúde, v. 5, n. 2, p. 36-50, 2020.

DI RENZO, L. et al. Eating habits and lifestyles changes during COVID-19 lockdown: an Italian survey. J Transl Med, v. 18, n. 229, p. 1-15, 2020.

DI RENZO, L. et al. Psychological Aspects and Eating Habits during COVID-19 Home Confinement: Results of EHLC-COVID-19 Italian Online Survey. Nutrients, v. 12, n. 7, p. 1-14, 2020.

FERNÁNDEZ-ARANDA, F. et al. Covid-19 and implications for eating disorders. Eur Eat Disord Ver, v. 28, n. 3, p. 239-245, 2020.

GOMES, R. Sexualidade masculina e saúde do homem: proposta para uma discussão. Ciência \& Saúde Coletiva, v. 8, n. 3, p. 825-829, 2003.

GOMES, R.; NASCIMENTO, EF.; ARAÚJO, FC. Por que os homens buscam menos os serviços de saúde do que as mulheres? As explicações de homens com baixa escolaridade e homens com ensino superior. Cad. Saúde Pública, v. 23, n. 3, p. 565- 574, 2007.

GÓRNICKA, M. et al. Dietary and Lifestyle Changes During COVID-19 and the Subsequent Lockdowns among Polish Adults: A Cross-Sectional Online Survey PLifeCOVID-19 Study. Nutrients, v. 12, n. 2324, p. 1-20, 2020.

HE, M. et al. Changes in Body Weight, Physical Activity, and Lifestyle During the Semi- lockdown Period After the Outbreak of COVID-19 in China: An Online Survey. Disaster Medicine and Public Health Preparedness. Disponível em

<https://www.ncbi.nlm.nih.gov/pmc/articles/PMC7385320/>. Acesso em 22 de Setembro de 2020.

KAKESHITA, IS. et al. Construção e Fidedignidade Teste-Reteste de Escalas de Silhuetas Brasileiras para Adultos e Crianças. Psicologia: Teoria e Pesquisa, v. 25, n. 2, p. 263-270, 2009.

MANTOVANI, LM. et al. Análise da alimentação e hidratação de praticantes de polo aquático do município de São Paulo. Revista Brasileira de Nutrição Esportiva, v. 11, n. 65, p. 570-576, 2017.

Organização Mundial da Saúde (OMS). Global Recommendations on Physical Activity for Health.Disponível em: 
<https://apps.who.int/iris/bitstream/handle/10665/44399/9789241599979_eng.pdf?se quence=1>. Acesso em 11 de Março de 2020.

PASCHOALICK, RC.; LACERDA, MR.; CENTA, ML. Gênero masculino e saúde.

Cogitare Enferm, v. 11, n. 1, p. 80-86, 2006.

REYES-OLAVARRÍA, D. et al. Positive and Negative Changes in Food Habits, Physical Activity Patterns, and Weight Status during COVID-19 Confinement: Associated Factors in the Chilean Population. Int. J. Environ. Res. Public Health, v. 17, n. 5431, p. 1-14, 2020.

RODRÍGUEZ-PÉREZ, C. et al. Changes in Dietary Behaviours during the COVID-19 Outbreak Confinement in the Spanish COVIDiet Study. Nutrients, v. 12, n. 1730, p. 1- 19, 2020.

RUIZ-ROSO, M. B. et al. Covid-19 Confinement and Changes of Adolescent's Dietary Trends in Italy, Spain, Chile, Colombia and Brazil. Nutrients, v. 12, n. 1807, p. 1-18, 2020.

SHEN, W. et al. A Humanities-Based Explanation for the Effects of Emotional Eating and Perceived Stress on Food Choice Motives during the COVID-19 Pandemic. Nutrients, v. 12, n. 2712, p. 1-18, 2020.

SRIVASTAV, AK.; SHARMA, N.; SAMUEL, AJ. Impact of Coronavirus disease-19 (COVID19) lockdown on physical activity and energy expenditure among physiotherapy professionals and students using web-based open E-survey sent through WhatsApp, Facebook and Instagram messengers. Clinical Epidemiology and Global Health. Disponível em <https://www.ncbi.nlm.nih.gov/pmc/articles/PMC7358172/>. Acesso em 22 de Setembro de 2020.

WEISSMAN, R. S.; BAUER, S.; THOMAS, J. J. Access to evidence-based care for eating disorders during the COVID-19 crisis. Int J Eat Disord, v. 53, p. 369-376, 2020.

ZACHARY, Z. et al. Self-quarantine and weight gain related risk factors during the COVID-19 pandemic. Obesity Research \& Clinical Practice, v. 14, p. 210-216, 2020. 